\title{
L'USO DELLA MODELLAZIONE COMPUTAZIONALE NELLE FUNZIONI CINEMATICHE CON MODELLUS
}

\section{ARTICOLO ORIGINALE}

CARDOSO, Kaio Felipe Nink¹, JUNIOR, Gilberto Nunes da Silva², JÚNIOR, João Hermano Torreiro de Carvalho ${ }^{3}$

CARDOSO, Kaio Felipe Nink. JUNIOR, Gilberto Nunes da Silva. JÚNIOR, João Hermano Torreiro de Carvalho. L'uso della modellazione computazionale nelle funzioni cinematiche con Modellus. Revista Científica Multidisciplinar Núcleo do Conhecimento. Anno 05, Ed. 03, Vol. 04, pp. 117-140. marzo 2020. ISSN: 24480959, Link di accesso: https://www.nucleodoconhecimento.com.br/formazioneit/cinematiche-con-modellus,

DOI:

10.32749/nucleodoconhecimento.com.br/formazione-it/cinematiche-con-modellus

\section{RIEPILOGO}

L'applicazione della fisica è ancora una sfida per i mezzi di insegnamento, basati su metodi tradizionali, in grado di escludere altri mezzi più moderni e che fanno un maggiore uso delle tecnologie educative. Abbiamo fatto uso del programma di modellazione computerizzazione, Modellus, focalizzatoprincipalmente sulle applicazioni cinematiche, con due esercizi che coinvolgono i movimenti di Vertical Release e Oblique Release, poiché vengono sollevate difficoltà per quanto riguarda la comprensione, eventualmente generata dai metodi educativi esposti. Abbiamo applicato gli esercizi in un questionario con 15 studenti del $2^{\circ}$ Grado di Liceo dell'IFBA/Barreiras, come campione per l'analisi del modo in cui gli studenti valutano l'attuale insegnamento della Fisica e delle possibili esigenze delle nuove metodologie nell'educazione di questa Scienza.

\footnotetext{
${ }^{1}$ Laureato in Informatica, Tecnico Informatico.

2 Tecnico informatico.

${ }^{3}$ Master in educazione.
}

RC: 93729

Disponibile in: https://www.nucleodoconhecimento.com.br/formazione-it/cinematichecon-modellus 
Parole chiave: insegnamento della fisica, software, modellazione computazionale, valutazione degli studenti.

\section{INTRODUZIONE}

Nel bel mezzo di numerosi progressi tecnologici, molto deve essere discusso su come viene applicata l'istruzione, dal momento che gesso e lavagna sono ancora pesantemente utilizzati in classe e potrebbero non dare spazio ad attività più dinamiche. Per quanto riguarda l'aspetto qualitativo, abbiamo una scuola del XVIII secolo nel $21^{\circ}$ secolo (VALENTE apud LOPES; FEITOSA, 2009). Ciò diventa evidente quando si nota come il modello educativo non abbia presentato diverse trasformazioni per quanto riguarda la sua forma di applicazione.

Si nota che gli studenti dimostrano una certa difficoltà nell'apprendimento delle Scienze Esatte, come la fisica, per esempio. L'uso di metodi didattici tradizionali e l'assenza di moderni mezzi pedagogici sono ragioni di questo problema (SANTOS; ALVES, MORET, 2006). D'altra parte, è noto che molti ambienti didattici potrebbero non avere risorse sufficienti per migliorare l'insegnamento in classe, nonché un'adeguata formazione professionale.

Presentandosi come software libero, Modellus è un'applicazione focalizzata sulla modellazione computazionale delle funzioni applicate per l'insegnamento della cinematica sia nelle scienze matematiche che in scienze fisiche, consentendo all'utente di fare e rifare rappresentazioni, esplorandole sulle prospettive più diverse (VEIT; TEODORO, 2002). Un'altra caratteristica importante fornita da Modellus è la rappresentazione multipla, cioè l'utente può creare, vedere e interagire con le rappresentazioni analitiche, analogiche e grafiche di oggetti matematici (TEODORO apud ARAUJO, 2002).

Tra le materie lavorate negli ambienti educativi in Fisica sulla meccanica, possiamo entrare nello studio della cinematica, concentrandosi sui movimenti del Rilascio Verticale e delle Release Oblique. Diventa possibile applicarli con l'aiuto di 
Modellus attraverso esercizi comuni deitemi, poiché con mezzi didattici tradizionali potrebbe non affascinare l'interesse dello studente, ma con un miglioramento della tecnica software, secondo diversi autori nell'area dell'insegnamento della fisica, si può ottenere un altro risultato.

Al fine di una buona applicazione degli esercizi, è stato sviluppato un questionario per gli studenti delle scuole superiori presso l'Istituto Federale di Educazione, Scienza e Tecnologia di Bahia - Campus Barreiras dei corsi di livello Tecnico Integrato, al fine di confrontare le prestazioni dell'insegnamento in Scienze Matematiche e Fisica con e senza l'uso di Modellus.

\section{USO DI MODELLUS NELL'INSEGNAMENTO DI FISICA}

La fisica, normalmente, rappresenta una scienza di duro studio, che la maggior parte delle persone ha difficoltà a comprendere e interpretare, essendo lo studio dei fenomeni fisici che avvengono intorno a noi. La necessità dell'essere umano di comprendere l'ambiente che lo circonda e di spiegare i fenomeni naturali è la genesi della Fisica (RAMALHO JUNIOR; FERRARO; SOARES, 1998, p.2). Pertanto, è chiaro che la fisica è di fondamentale importanza per lo sviluppo umano, che secondo il fisico contemporaneo Michio Kaku, è ovunque, come ad esempio: nell'evoluzione delle tecnologie, dei mezzi di comunicazione e dei trasporti, tra gli altri.

La rilevanza di questo studio non è negata e dovrebbe essere insegnato come un modo per osservare il mondo e capirlo, tuttavia, il suo insegnamento è ancora oggi molto fuori tempo. Secondo la ricerca di Rosa, Perez e Drum (2009), i fisici intervistati hanno affermato che c'è l'importanza degli esperimenti fisici, come modo per dimostrare la realtà, all'interpretazione dello studente, che sarebbe il modo più appropriato di insegnare. Da questo, ci siamo resi conto che l'educazione fisica non è molto attraente, presentando molti calcoli e visualizzazione logica dei sistemi, che capiscono la mancanza di interesse per esso.

RC: 93729

Disponibile in: https://www.nucleodoconhecimento.com.br/formazione-it/cinematichecon-modellus 
Molto spesso, la mancanza di diverse tecniche metodologiche è responsabile di un alto numero di "analfabeti" in fisica nel mondo. La ricerca di Teixeira, Júnior e Golfette (2004), osservata nella figura 1, ci mostra gran parte della realtà di questa formazione in Brasile, affrontando questi problemi di insegnamento: sono necessarie la mancanza di preparazione degli studenti (una precedente conoscenza della matematica e una buona interpretazione dei sistemi), un vecchio sistema educativo, la mancanza di materiale didattico attraente, infrastrutture e formazione degli insegnanti.

Figura 1 - Difficoltà nell'insegnamento della fisica (TEIXEIRA; JÚNIOR; GOLFETTE, 2004)

\section{Quais os grandes desafios que o Brasil deve enfrentar para melhorar o ensino de Física? (Principais respostas)}

Melhorar políticas em C \& T Repensar os conteúdos Melhorar a condiçẫo dos alunos Melhorar as políticas em Educaçẫo

Melhorar os materiais de apoio Melhorar a infraestrutura da escola Melhorar a formaçẫo dos professores

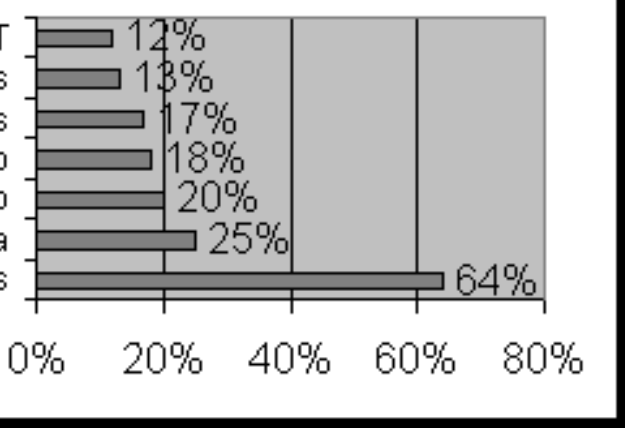

"Nelle narrazioni degli insegnanti, evidenziano la loro visione della fisica, perché per alcuni (coloro che condividono l'impossibilità di questo insegnamento), la fisica si riduce ai calcoli, alle situazioni problematiche in cui è richiesto un ragionamento matematico e algebrico complesso; questa situazione, a loro avviso, è ancora in fase di sviluppo nello studente dei voti iniziali, non permettendogli di contemplarla in questa fase scolastica. (ROSA; PEREZ; DRUM, 2009, p. 362)

Secondo il grafico della figura 1, la formazione degli insegnanti è una questione da affrontare, tuttavia, anche se viene evidenziata circa tre volte di più dei materiali di sostegno. Si percepisce che questi, se appropriati di migliori tattiche didattiche, aiuteranno anche a ridurre il livello di formazione degli insegnanti, poiché agirà a tutti

RC: 93729

Disponibile in: https://www.nucleodoconhecimento.com.br/formazione-it/cinematichecon-modellus 
i livelli di istruzione. Forme alternative sarebbero, Internet come supporto tecnico e l'aumento delle procedure sperimentali che inducono lo studente alla visualizzazione, tuttavia, molti sistemi studiati non sono possibili per riprodurli perché lavorano con sistemi conservativi (nel vuoto), quindi, una possibilità legata a questo interrogatorio, sarebbe l'uso di software di modellazione computerizzata, un esempio è il Modellus.

Secondo Aliprandini (2009), forme alternative di insegnamento della Fisica, attraverso simulazioni o nella modellazione dell'ambiente reale, consentono il cambiamento degli standard didattici tradizionali (ALIPRANDINI; SCHUHMACHER; SANTOS, 2009).

Modellus è un programma educativo di modellazione computazionale che permette la creazione, la simulazione di fenomeni fisici e matematici, utilizzati nell'insegnamento delle scienze esatte. Permette di creare, preparare e risolvere un problema, utilizzando la rappresentazione matematica del sistema per simularlo, manipolando così le variabili (tempo, distanza, ecc.) e osservando come si verifica il fenomeno fisico. L'idea è quella di usarlo come un modo di interpretare i problemi, osservando ciò che ogni grandezza esercita di influenza, indipendentemente dai calcoli.

"Il programma può essere considerato un'animazione interattiva e considerato uno strumento computazionale che aiuta la costruzione della conoscenza e può essere utilizzato per riformulare la conoscenza attraverso significati chiari, stabili e differenziati precedentemente esistenti nella struttura cognitiva dello studente". (ALIPRANDINI; SCHUHMACHER; SANTOS, 2009, p. 1374)

Ciò che si intende quando si utilizza il software nell'insegnamento della fisica è che gli studenti di fisica sappiano utilizzare le conoscenze scientifiche acquisite nel processo decisionale, dallo sviluppo del loro intelletto in due modi, esplorativo ed espressivo, secondo Aliprandini, Schuhmacher e Santos (2009). La modalità esplorativa riguarda la localizzazione di funzioni analitiche, analogiche e grafiche nel programma, che sviluppa conoscenze matematiche per associazione, oltre all'esplorazione dei sistemi di altre persone; e la modalità espressiva, come un modo per dimostrare la creatività, creando i modelli degli utenti.

RC: 93729

Disponibile in: https://www.nucleodoconhecimento.com.br/formazione-it/cinematichecon-modellus 
Per Veit e Teodoro (2002), Modellus consente "l'apprendimento costruttivista", che avviene dalla definizione delle idee, con benefici a livello cognitivo. Questo processo è dovuto al fatto che l'utilizzo del software non è affatto banale, essendo un lento processo di apprendimento che richiede un pensiero architettonico per costruire le simulazioni, avendo la costruzione non solo cognitiva, ma anche personale, raggiungendo l'obiettivo del sviluppo della visione del mondo degli studenti sulla base delle conoscenze scientifiche acquisite.

Un aspetto che mostra l'uso di Modellus in campo educativo è che viene utilizzato in istituzioni di tutto il mondo, come il progetto Institute of Physics nel Regno Unito. II programma ha ottenuto il riconoscimento mondiale nel 1996 con il premio "1996 Software Contest of the Journal Computer in Physics" e nel 1998 con il $1^{\circ}$ posto al National Microsoft Software Contest, 1998 a Lisbona (VEIT e TEODORO, 2002). La Figura 2 mostra il software Modellus sotto forma di mappa concettuale. In breve, la modellazione computazionale, legata alla scienza della natura fisica, rende la relazione tra le equazioni e le funzioni della fisica (algoritmo) che descrivono il fenomeno osservato (teoria) e la sua applicazione attraverso la simulazione al computer, all'interno di un linguaggio comune all'utente, che ovvero, senza la necessità di imparare a programmare in una lingua diversa da quella già vista in classe (figura 3). 
Figura 2 - Usi per il software (VEIT; TEODORO, 2002)

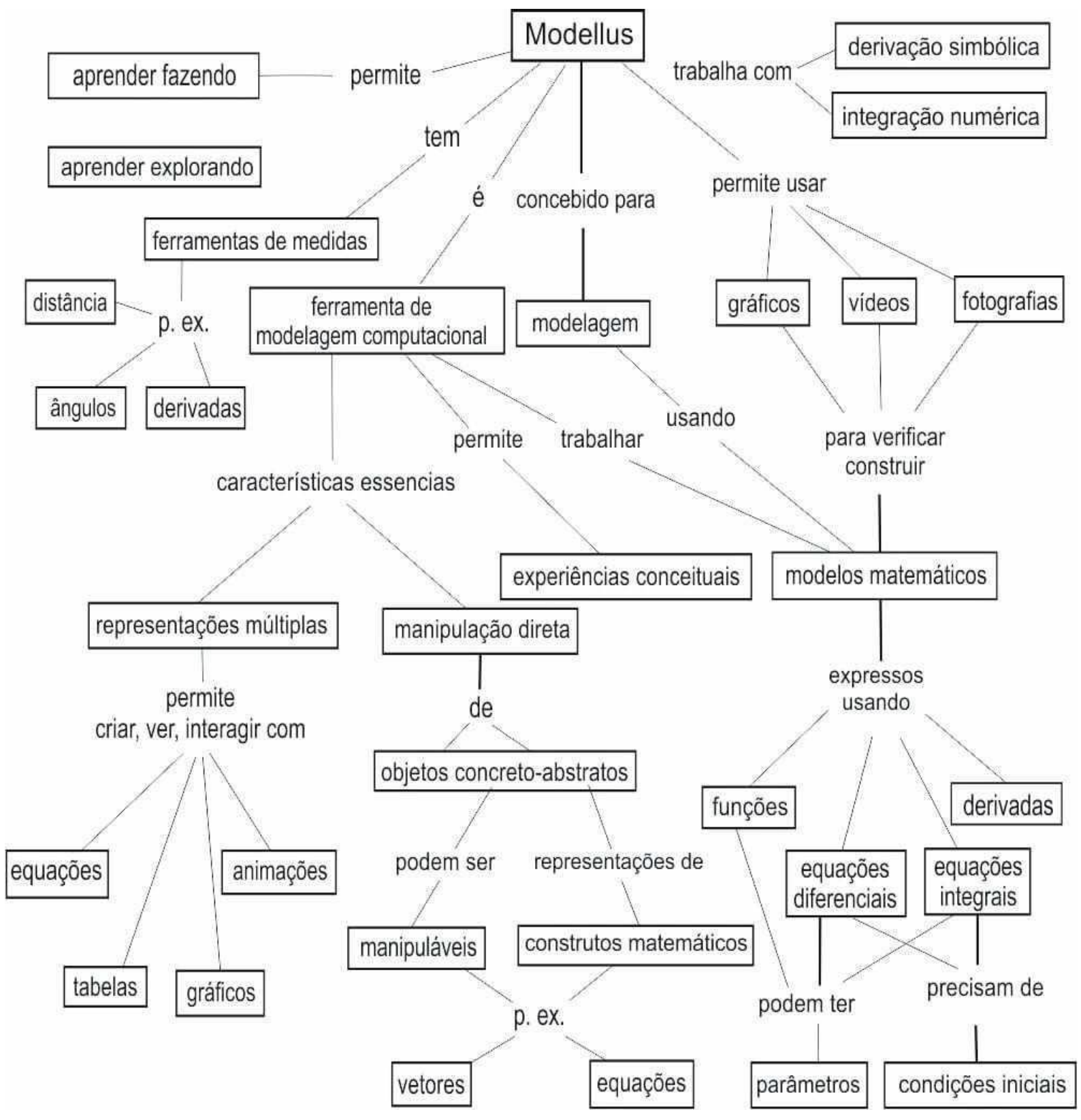

RC: 93729

Disponibile in: https://www.nucleodoconhecimento.com.br/formazione-it/cinematichecon-modellus 
Figura 3 - Relazione di conoscenza nella modellazione computazionale (Autori, 2017)

\section{Arquitetura}

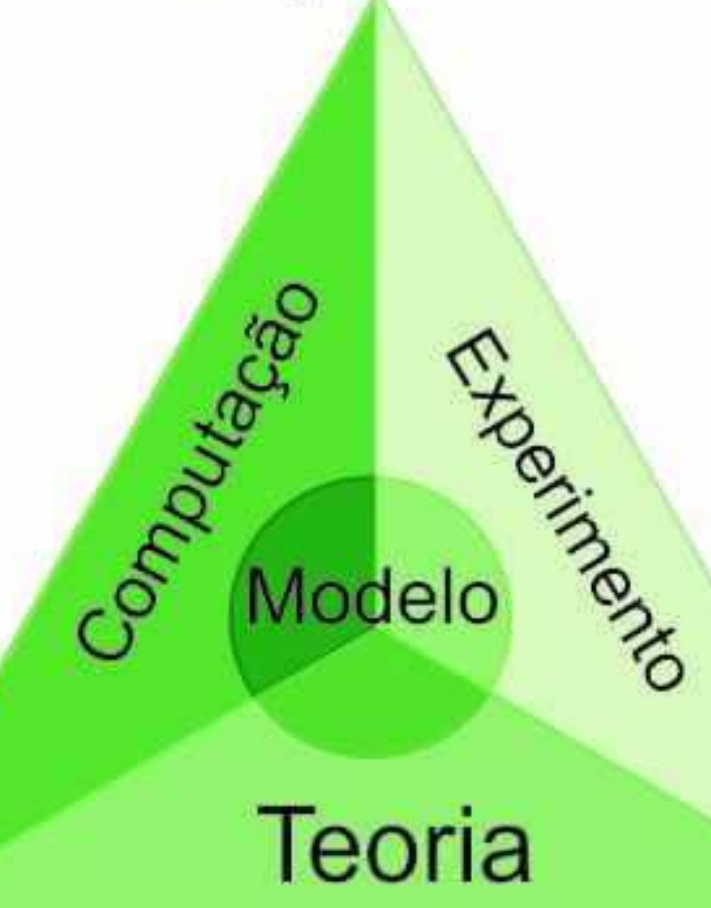

\section{Aplicação}

\section{Algoritmo}

\section{MATERIALI E METODI}

\subsection{ESERCIZI COME ESEMPIO CON MODELLUS}

La cinematica è la parte della meccanica che descrive i movimenti, cercando di determinare la posizione, la velocità e l'accelerazione di un corpo in ogni istante. (RAMALHO JUNIOR; FERRARO; SOARES, 1998). Negli ambienti educativi, ci sono le applicazioni, spesso teoriche ed espositive, dei temi precedentemente affrontati. Questo è stato lavorato con due esercizi teorici presi da un libro di testo del liceo:

Ex. 01. (Lancio verticale) Due mobili A e B vengono lanciati verticalmente verso l'alto, con la stessa velocità iniziale di $15 \mathrm{~m} / \mathrm{s}^{2}$, dallo stesso punto. Mobile $A$ viene rilasciato 
istantaneamente $\mathrm{t}=0$ s e mobile $\mathrm{B}$ viene rilasciato 2 s più tardi. Determinare, dal punto di lancio, la posizione e il momento della riunione dei mobili. Prendere $\mathrm{g}=10 \mathrm{~m} / \mathrm{s}^{2} \mathrm{e}$ ignorare la resistenza all'aria.

Ex. 02. (Rilascio obliquo) Un corpo viene rilasciato obliquamente nel vuoto con velocità iniziale $v 0=100 \mathrm{~m} / \mathrm{s}$, in una direzione che forma con l'orizzontale un angolo $\theta$ tale che $\operatorname{sen} \theta=0,8$ e $\cos \theta=0,6$. Adottando $g=10 \mathrm{~m} / \mathrm{s}^{2}$, determinare:

— i moduli dei componenti orizzontali e verticali della velocità al momento del lancio;

l'istante in cui il corpo raggiunge il punto più alto della traiettoria;

l'altezza massima raggiunta dal corpo;

la portata del lancio;

\subsection{VALUTAZIONE DEGLI STUDENTI DI FISICA E FISICA TRADIZIONALE CON L'USO DI MODELLUS}

Approfondendo in ambito educativo, è possibile divergere $\mathrm{i}$ metodi didattici tradizionali esposti da metodi significativi. Una prima deve comprendere l'approccio tradizionale:

"Come sappiamo, l'adulto, nella concezione tradizionale, è considerato come l'uomo finito, "pronto" e lo studente un "adulto in miniatura", che deve essere aggiornato. L'insegnamento, in tutte le sue forme, in questo approccio, sarà incentrato sul maestro. Questo tipo di insegnamento si rivolge a ciò che è esterno allo studente: il programma, le discipline, il docente. Lo studente esegue solo prescrizioni fissate da autorità esterne" (MIZUKAMI, 1986, p. 8)

Questo processo è ampiamente visto nelle aule, ad esempio, una lezione di fisica espositiva con un insegnante che esegue esercizi alla lavagna può implicare i risultati ottenuti sulle prestazioni degli studenti. Secondo Pelizzari, Kriegl, Baron, Finck e Dorocinski (2002, p 38), sulla base dello studio di Ausubel, abbiamo un'altra prospettiva, dall'approccio significativo: 
"L'apprendimento è molto più significativo in quanto i nuovi contenuti sono incorporati nelle strutture della conoscenza di uno studente e acquisiscono significato per loro dalla relazione con le loro conoscenze precedenti. Al contrario, diventa meccanico o ripetitivo, poiché si produce meno questa incorporazione e attribuzione di significato, e il nuovo contenuto inizia ad essere immagazzinato da solo o attraverso associazioni arbitrarie nella struttura cognitiva".

Utilizzando le idee di Santos, Alves e Moret e Santos (2006), abbiamo utilizzato un questionario per valutare la conoscenza degli studenti che hanno partecipato. Le persone coinvolte costituiscono il Corso Tecnico di Liceo, per un totale di 15 studenti. La prima fase è seguita da domande all'insaputa degli studenti su Modellus, come modo per valutare i metodi di istruzione in classe.

1. Qual è il tuo rapporto con la disciplina della fisica? (Non mi piace; Mi piace un po'; Mi piace. Mi piace molto)

2. Hai avuto difficoltà nello studio del rilascio verticale e del rilascio obliquo? (Sì; Poco; No)

3. Esercizio di registrazione verticale proposto

4. Esercizio di lancio obliquo proposto

5. Crede che i metodi di insegnamento tradizionali possano influenzare la mancanza di interesse della disciplina? (No; Un po'; Qualunque cosa faccia; Sì, non ho intenzione di Abbastanza)

6. Crede che l'uso del softwares possa contribuire alla comprensione sia della disciplina della Matematica che della Fisica? (No; Un po'; Qualunque cosa faccia; Sì, non ho intenzione di Abbastanza)

Successivamente, è stata fatta una presentazione espositiva sul Modellus, evidenziandone le applicazioni e i contributi all'insegnamento della Fisica e della Matematica. Dopo questa introduzione, gli esercizi utilizzati nel questionario precedente sono stati applicati nel software, analizzando le informazioni disposte nella loro simulazione. Si è concluso con una seconda fase consistente in un questionario sull'uso di Modellus una volta presentato: 
1. L'uso della modellazione computazionale ha facilitato la comprensione degli esercizi proposti? (No; Un po'; Qualunque cosa faccia; Sì, non ho intenzione di Abbastanza)

Hai avuto difficoltà nel modo in cui il programma presenta i suoi risultati? (No; Un po'; Qualunque cosa; Sì, non ho intenzione di Abbastanza)

Crede che l'uso di softwares come Modellus potrebbe essere utilizzato in classe per contribuire all'insegnamento della Fisica? (No; Qualunque cosa; Sì)

Qual è la sua ultima considerazione del lavoro svolto? (Domanda discorsiva)

\section{RISULTATI E DISCUSSIONI}

\subsection{ESERCIZI APPLICATI IN MODELLUS}

Nell'esercizio di lancio verticale 1, sono richiesti la posizione istantanea e di incontro dei mobili $\mathrm{A}$ e $\mathrm{B}$.Considerando che $\mathrm{sA}=\mathrm{sB}$, è facile utilizzare la funzione spazio orario per trovare l'istante della riunione $(\mathrm{t}=2,5 \mathrm{~s})$ e successivamente trovare la posizione ( $\mathrm{s} A=\mathrm{sB}=6,25 \mathrm{~m})$.

Nell'esercizio 2 di Oblique Launch, il modulo di velocità è ricercato nell'istante iniziale del lancio, sia orizzontalmente $(\mathrm{Vx}=60 \mathrm{~m} / \mathrm{s})$ che verticalmente $(\mathrm{V} 0 \mathrm{y}=80 \mathrm{~m} / \mathrm{s})$, così come l'istante del corpo nel punto più alto $(\mathrm{t}=8 \mathrm{~s})$, l'altezza massima della traiettoria $(\mathrm{H}=320 \mathrm{~m})$ e la gamma di lancio $(\mathrm{A}=960 \mathrm{~m})$. Utilizzando le operazioni matematiche del movimento orizzontale, è possibile trovare i rispettivi risultati.

Entrambi gli esercizi si trovano facilmente nei libri di testo o su Internet stesso con le loro risoluzioni più varie. Quello che può sembrare un semplice problema per un dato studente può causare qualche disagio per altri, il che è un punto di problematizzazione su come i metodi tradizionali possono influenzare tali risultati. 
L'uso della modellazione computazionale viene fornito come prospettiva per cambiare questo scenario. Avendo lo sviluppo dei problemi precedenti nel software Modellus, è possibile ottenere un'ampiezza nei risultati, come mostrato nella figura 4 :

Figura 4 - Modello matematico per l'esercizio di lancio verticale proposto in Modellus (Autori, 2017)

\begin{tabular}{|l|}
\hline Modelo Matemático \\
\hline$x=v x \times t$ \\
$y=V y O \times t-\frac{g}{2} \times t^{2}$ \\
$g=10$ \\
$v O=100$ \\
$V y O=v O \times 0.8$ \\
$V x=v O \times 0.6$ \\
$v y=V y O-g \times t$ \\
\hline Parâmetros Condigões inicais \\
\hline
\end{tabular}


Figura 5 - Simulazione dell'esercizio di rilascio verticale proposto in Modellus (Autori, 2017)

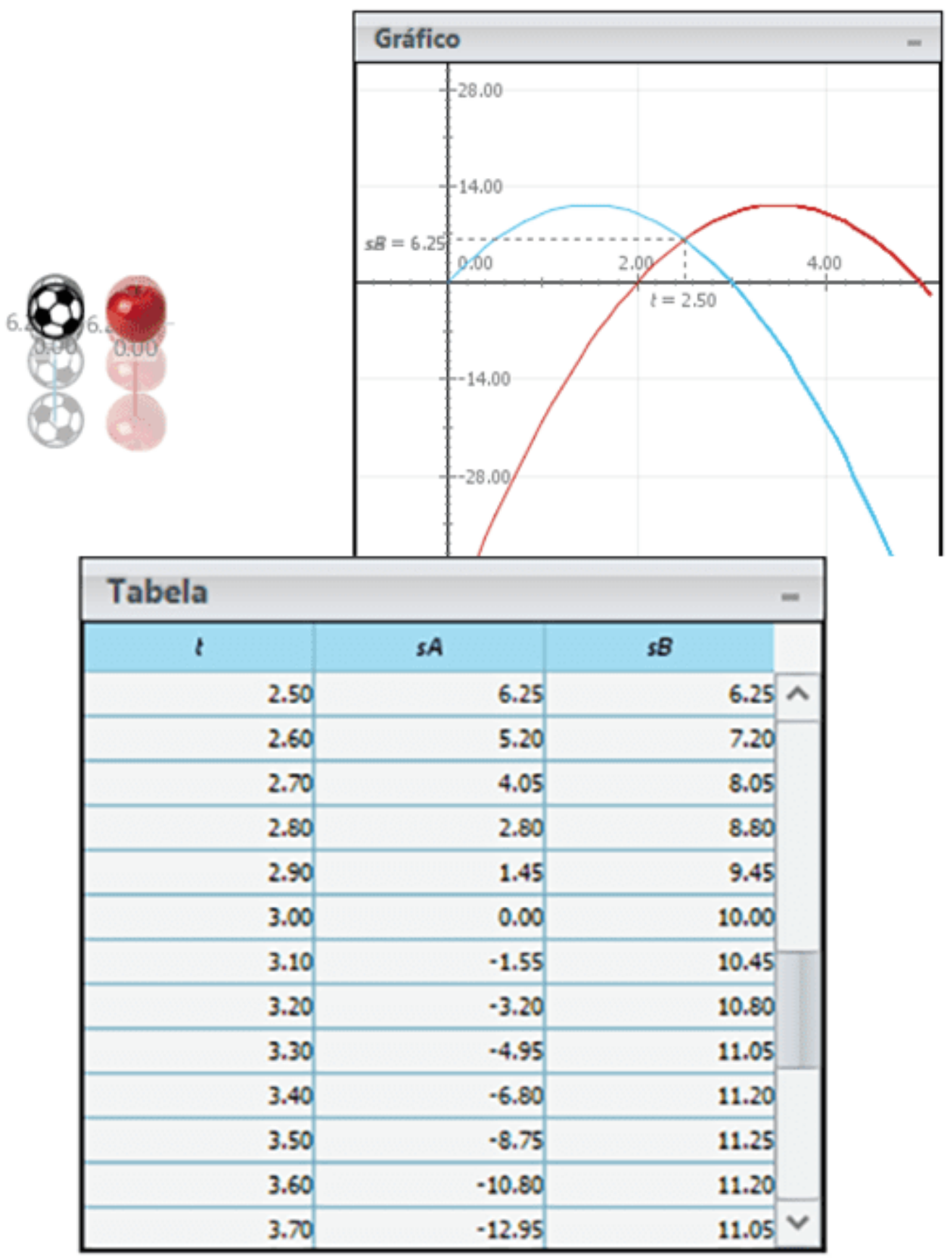

II software dipenderà dalla descrizione delle funzioni matematiche da applicare, rendendo possibile ottenere il risultato attraverso animazioni, grafici e tabelle. Entrambi i corpi obbediscono alle loro funzioni. La figura 5 mostra l'istante dei corpi,

RC: 93729

Disponibile in: https://www.nucleodoconhecimento.com.br/formazione-it/cinematichecon-modellus 
visto attraverso il rispettivo grafico e tabella. Dalla tabella, l'istante (t=2,5 s) mostra il momento dell'incontro in cui $\mathrm{Sa}=\mathrm{Sb}$, così come il grafico delle posizioni in funzione del tempo, secondo il punto di incontro.

Per la situazione di problema successiva, come mostrato nella figura 6, c'è stata anche un'applicazione attraverso la modellazione computazionale

Figura 6 - Modello matematico all'esercizio proposto di Oblique Release in the Modellus (Autori, 2017)

\begin{tabular}{|l||}
\hline Modelo Matemático \\
\hline$S A=s O+v O \times t-a \times t^{2}$ \\
$S O=0$ \\
$v O=15$ \\
$a=5$ \\
$S B=s O+v O \times(t-2)-a \times(t-2)^{2}$ \\
$S A=S B$ \\
\hline Parâmetros Condigóes lniciais \\
\hline
\end{tabular}


Figura 7 - Simulazione dell'esercizio di rilascio obliquo proposto nel Modellus (Autori, 2017)

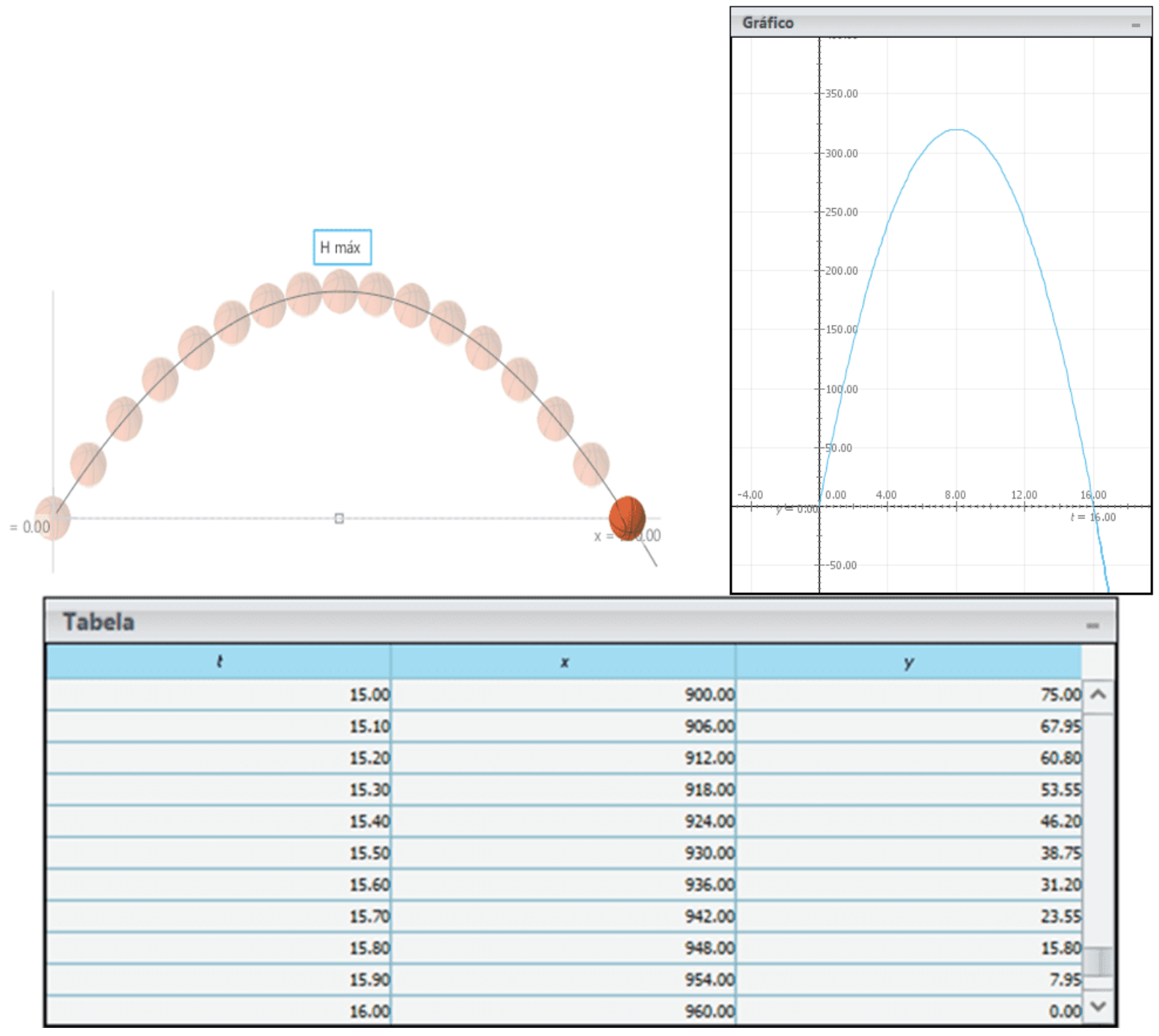

Dalla simulazione, come mostrato nella figura 7 , è possibile analizzare i risultati messi in discussione, come l'intervallo del lancio $A=960 \mathrm{~m}$, o l'istante t nel punto più alto come $8 \mathrm{~s}$. Alcuni valori possono essere facilmente ottenuti, ad esempio, per mezzo di un grafico nel programma, come l'altezza massima $\mathrm{H}=320 \mathrm{~m}$, vista dall'asse $Y$.

Vale la pena ricordare che il programma ti consente di aumentare le tue simulazioni, come cambiare la particella per un pallone da calcio o un basket, aggiungere

Disponibile in: https://www.nucleodoconhecimento.com.br/formazione-it/cinematichecon-modellus 
immagini sullo sfondo dell'animazione e cambiare i colori nel grafico e i dati che avranno, consentendo così una maggiore interazione dell'utente con il tuo ambiente di lavoro.

\subsection{RISULTATI OTTENUTI NELLA VALUTAZIONE DEGLI STUDENTI}

Attraverso il questionario di valutazione, sono state ottenute le prospettive prima dell'introduzione di Modellus e successivamente. È iniziato con le precedenti domande della prima fase, ma come forma valutativa dell'attuale insegnamento della Fisica dal punto di vista dello studente:

Figura 8 - Grafico della domanda 1 della prima fase (Autori, 2017)

1. Qual è il tuo rapporto con la disciplina fisica?

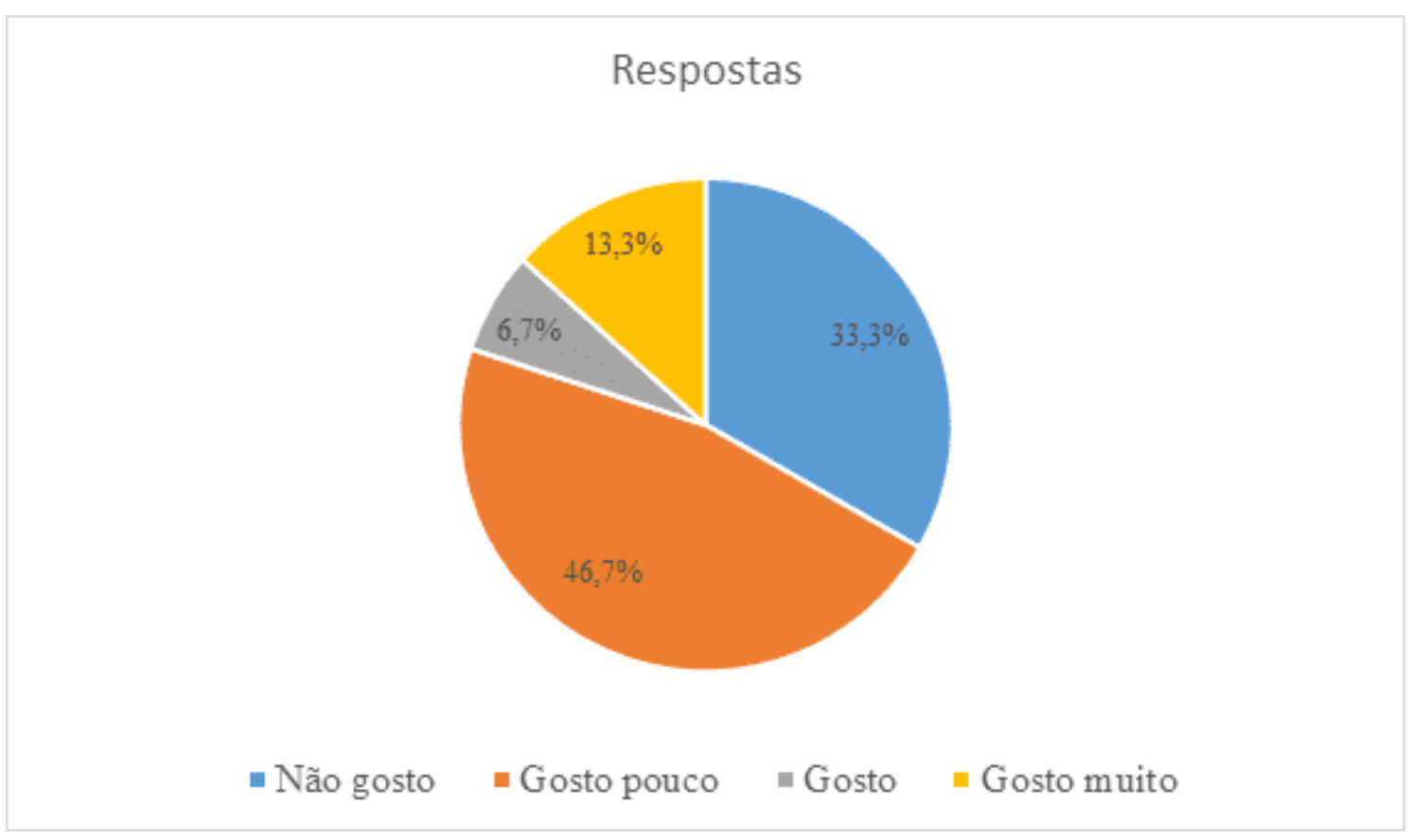

RC: 93729

Disponibile in: https://www.nucleodoconhecimento.com.br/formazione-it/cinematichecon-modellus 
Figura 9 - Grafico della domanda 2 della prima fase (Autori, 2017)

2. Hai avuto difficoltà nello studio del rilascio verticale e del rilascio obliquo?

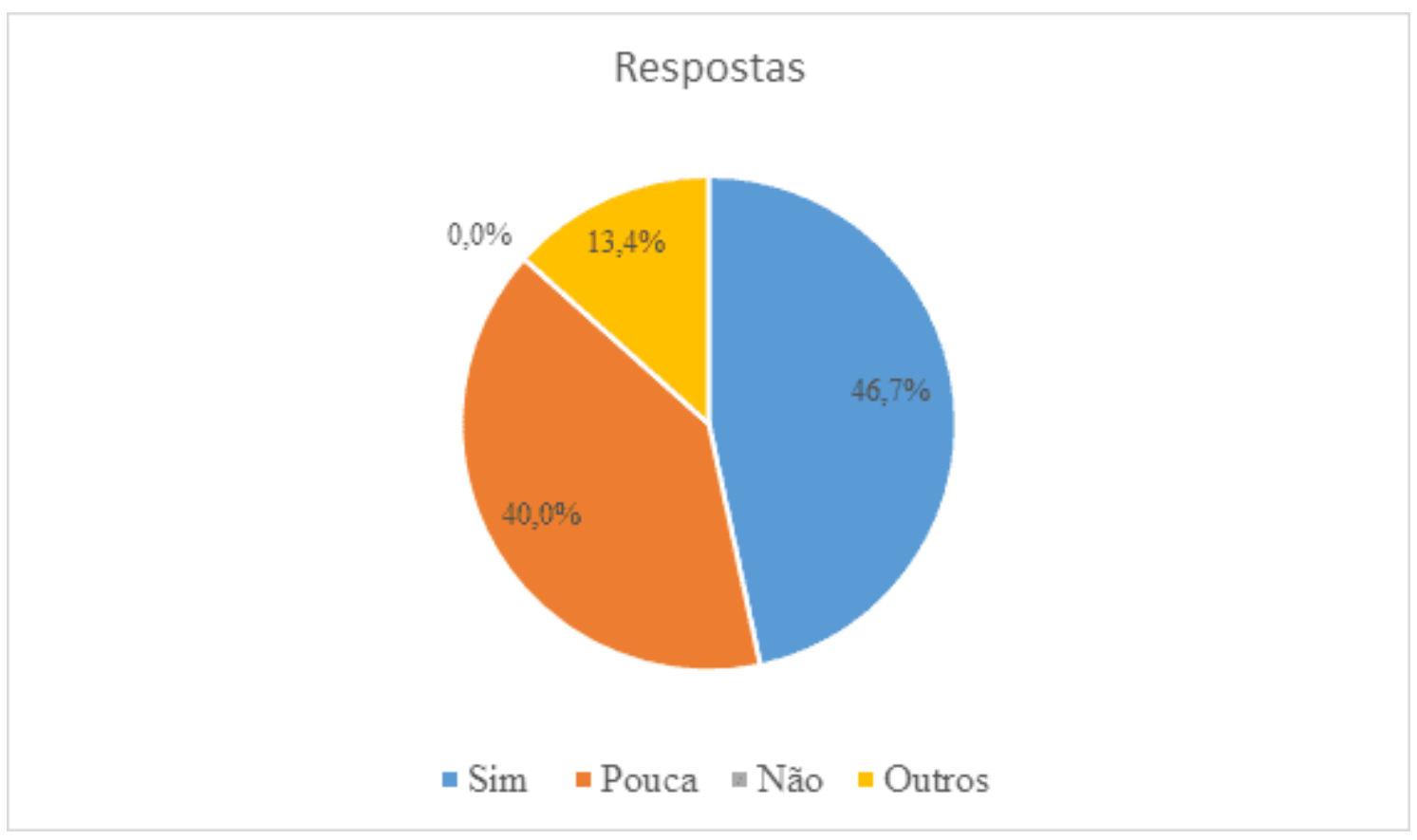

RC: 93729

Disponibile in: https://www.nucleodoconhecimento.com.br/formazione-it/cinematichecon-modellus 
Figura 10 - Domanda 3 Grafico della prima fase (Autori, 2017)

3. Ex. 01. (Lancio verticale) Due mobili $A$ e $B$ vengono lanciati verticalmente verso l'alto, con la stessa velocità iniziale di $15 \mathrm{~m} / \mathrm{s}^{2}$, dallo stesso punto. Mobile $A$ viene rilasciato immediatamente $t=0 \mathrm{~s}$ e il cellulare $B$ viene rilasciato $2 \mathrm{~s}$ più tardi. Determinare, dal punto di lancio, la posizione e il momento della riunione dei mobili. Prendere $\mathrm{g}=10 \mathrm{~m} / \mathrm{s}^{2}$ e ignorare la resistenza all'aria.

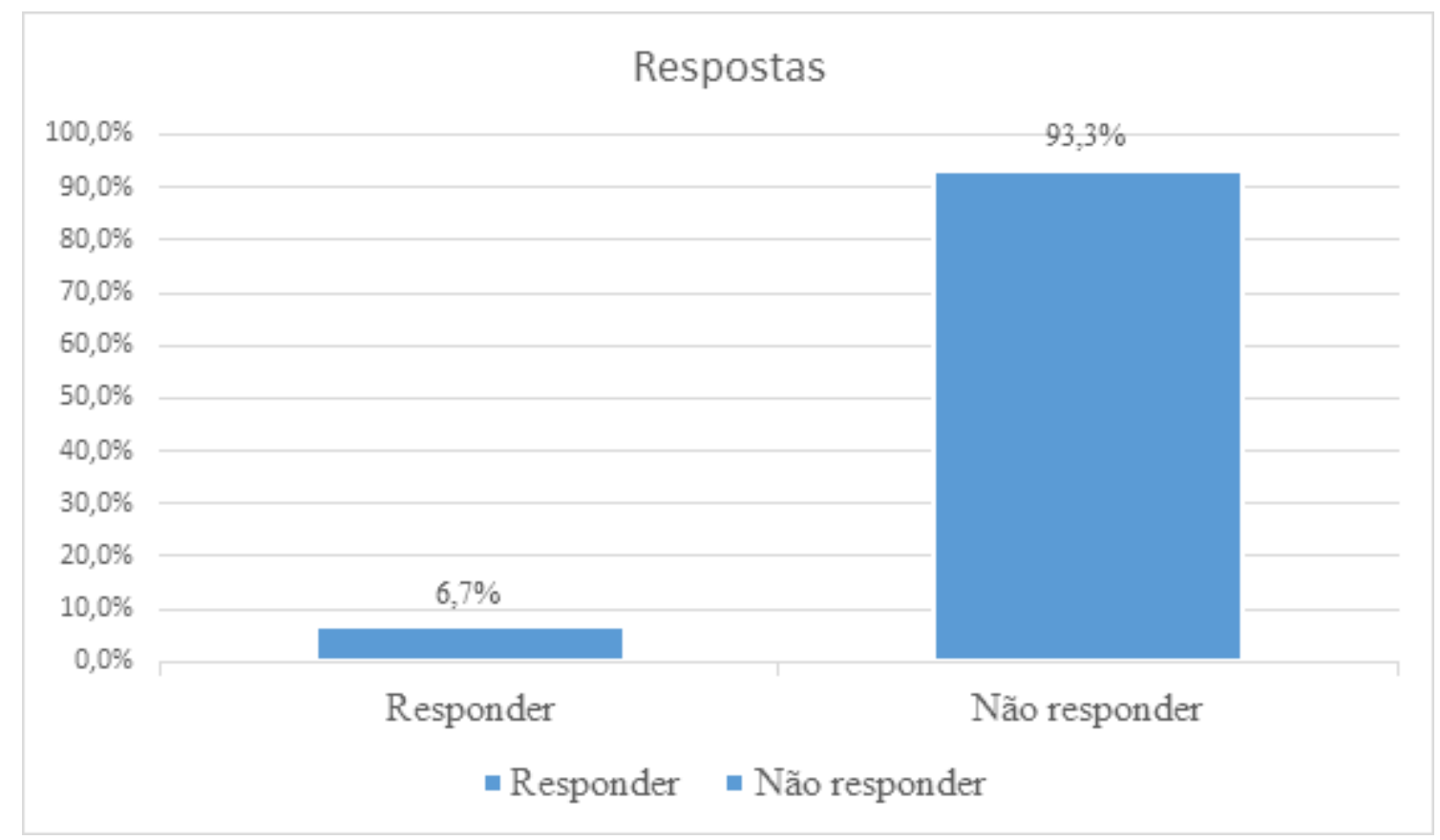

RC: 93729

Disponibile in: https://www.nucleodoconhecimento.com.br/formazione-it/cinematichecon-modellus 
Figura 11 - Domanda 4 Grafico della prima fase (Autori, 2017)

4. Ex. 02. (Rilascio obliquo) Un corpo viene rilasciato obliquamente nel vuoto con velocità iniziale $\mathrm{v} 0=100 \mathrm{~m} / \mathrm{s}$, in una direzione che forma con l'orizzontale un angolo $\theta$ tale che $\operatorname{sen} \theta=0,8$ e $\cos \theta=0,6$. Adottando $g=10 \mathrm{~m} / \mathrm{s}^{2}$, determinare: a) i moduli delle componenti orizzontali e verticali della velocità al momento del rilascio; b) l'istante in cui il corpo raggiunge il punto più alto della traiettoria; c) l'altezza massima raggiunta dal corpo; $\mathrm{d}$ la portata del lancio.

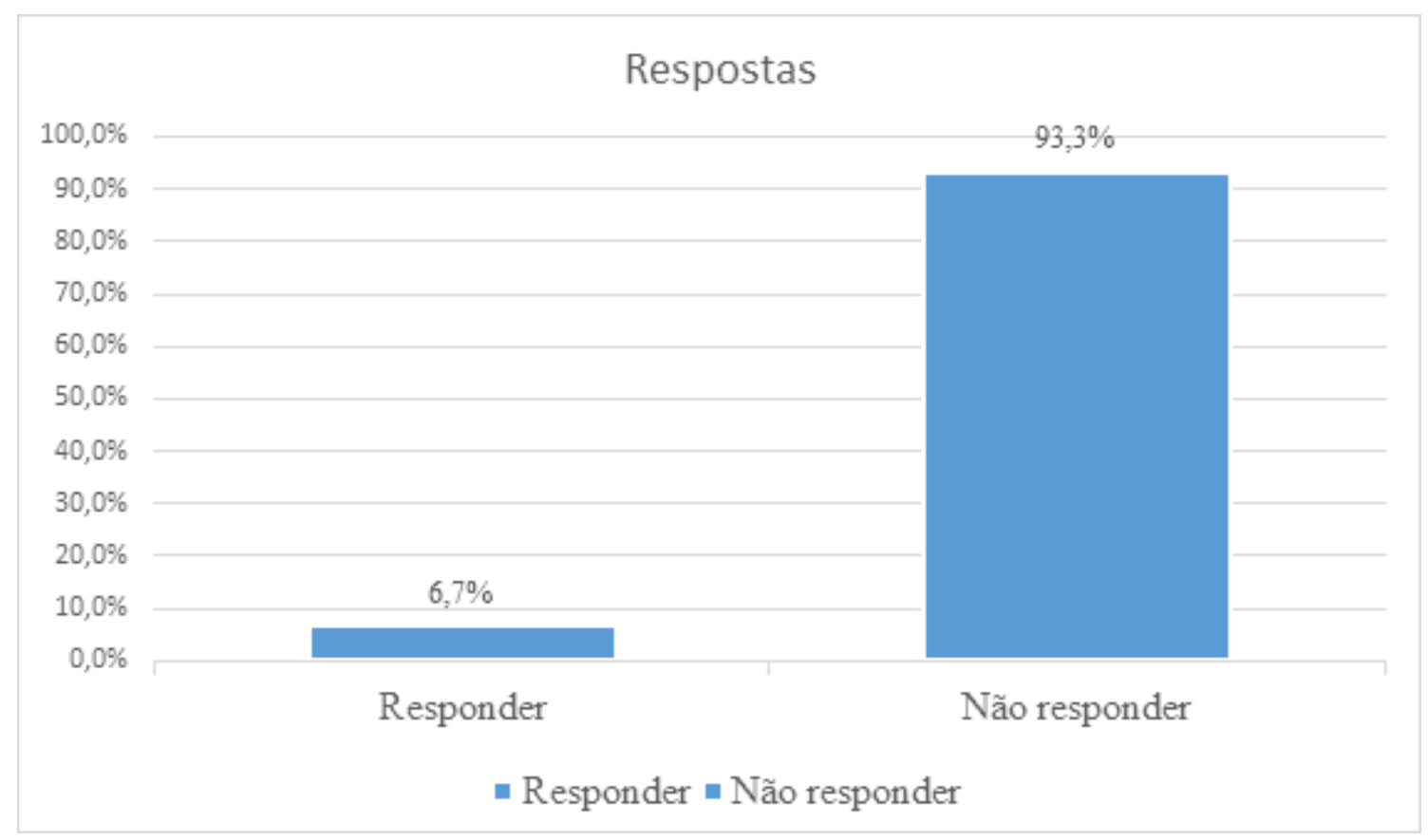

RC: 93729

Disponibile in: https://www.nucleodoconhecimento.com.br/formazione-it/cinematichecon-modellus 
Figura 12 - Domanda 5 Grafico della prima fase (Autori, 2017)

5. Crede che i metodi di insegnamento tradizionali possano influenzare la mancanza di interesse per la disciplina?

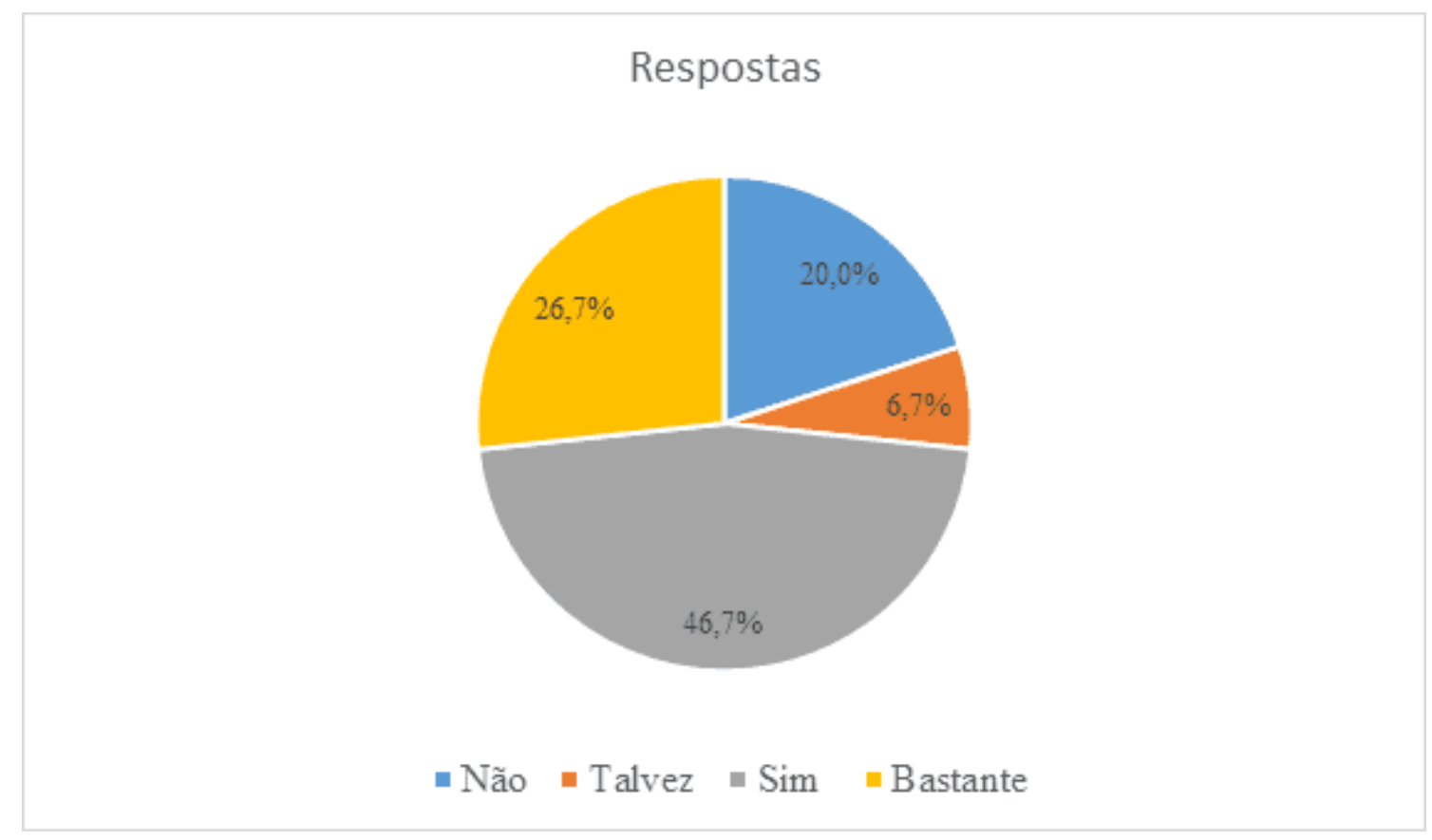

RC: 93729

Disponibile in: https://www.nucleodoconhecimento.com.br/formazione-it/cinematichecon-modellus 
Figura 13 - Domanda 6 Grafico della prima fase (Autori, 2017)

6. Credete che l'uso di altri metodi didattici (software, lezioni pratiche, ecc.) può contribuire alla comprensione sia della disciplina della Matematica che della Fisica?

\section{Respostas}

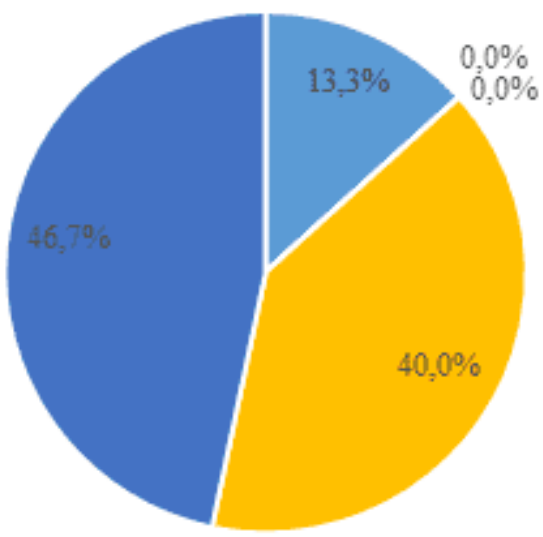

- Não - Um pouco $\quad$ "Tanto faz $\|$ Sim - Bastante

In generale, possiamo analizzare all'inizio, secondo il grafico nella figura 8, che la maggior parte dei partecipanti non ha un ampio interesse per la fisica: una parte maggioritaria ha presentato che gli piaceva la fisica come disciplina o che non gli piaceva, un fattore presente anche nella seconda domanda, secondo il grafico nella figura 10 , in cui la maggioranza sentiva difficoltà nell'apprendimento dei temi funzionava o sentiva poca difficoltà. C'è l'esempio di un partecipante che ha studiato i contenuti da solo.

In relazione ai due esercizi lavorati, si nota che solo un partecipante ha proposto di risolvere le domande, mentre gli altri le hanno lasciate vuote, secondo le figure $10 \mathrm{e}$ 11. È possibile mettere in relazione questo fattore con la mancanza di interesse per $i$ due temi disposti, nell'attività valutativa stessa o per la difficoltà stessa nell'argomento. Per Veit e Teodoro (2002), "in pratica, la Fisica rappresenta per lo studente, per la maggior parte, una disciplina molto difficile, in cui è necessario 
decorare formule la cui origine e scopo sono sconosciuti". Indipendentemente dal motivo in questione, è importante essere sicuri che gli studenti siano più interessati o possano ridurre al minimo le loro difficoltà in modo ampio.

Le ultime due domande presentano come una panoramica che verrà da una visione del partecipante stesso, ma in un'ampia visione dei metodi che compongono l'insegnamento della scienza oggi. La maggior parte dei partecipanti ha sottolineato, secondo la figura 12, che la forma tradizionale di insegnamento può influenzare l'interesse per la disciplina, oltre a ciò altre metodologie potrebbero essere più utilizzate, secondo la figura 13. Possiamo allearsi secondo Lima e Vasconcelos (2006, p. 399) sulla base dello studio di Krasilchik (2004, p. 184):

\footnotetext{
"L'insegnante, per mancanza di fiducia in se stesso, preparazione o mercismo, si limita a presentare agli studenti, con modifiche minime, il materiale precedentemente preparato da autori che sono accettati come autorità. Supportato da materiale progettato da altri e prodotto industrialmente, l'insegnante rinuncia alla sua autonomia e libertà, diventando semplicemente un tecnico.
}

Così si parte da una visione in cui gli studenti non si sentono ampiamente soddisfatti del modo in cui imparano o imparano - essendo così creduti - disciplina. Un corso di esibizione monotono con un'enorme quantità di calcoli sarebbe un esempio.

Ciò segue con la seconda parte della valutazione tramite questionario, costituendo un altro parere su Modellus e le sue applicazioni. Dopo la presentazione dello stesso, ai partecipanti è stato chiesto di valutarlo, rendendo possibile analizzare se i partecipanti si sentivano motivati o meno alla tecnologia:

RC: 93729

Disponibile in: https://www.nucleodoconhecimento.com.br/formazione-it/cinematichecon-modellus 
Figura 14 - Domanda 1 della seconda fase (Autori, 2017)

1. L'uso della modellazione computazionale ha facilitato la comprensione degli esercizi proposti?

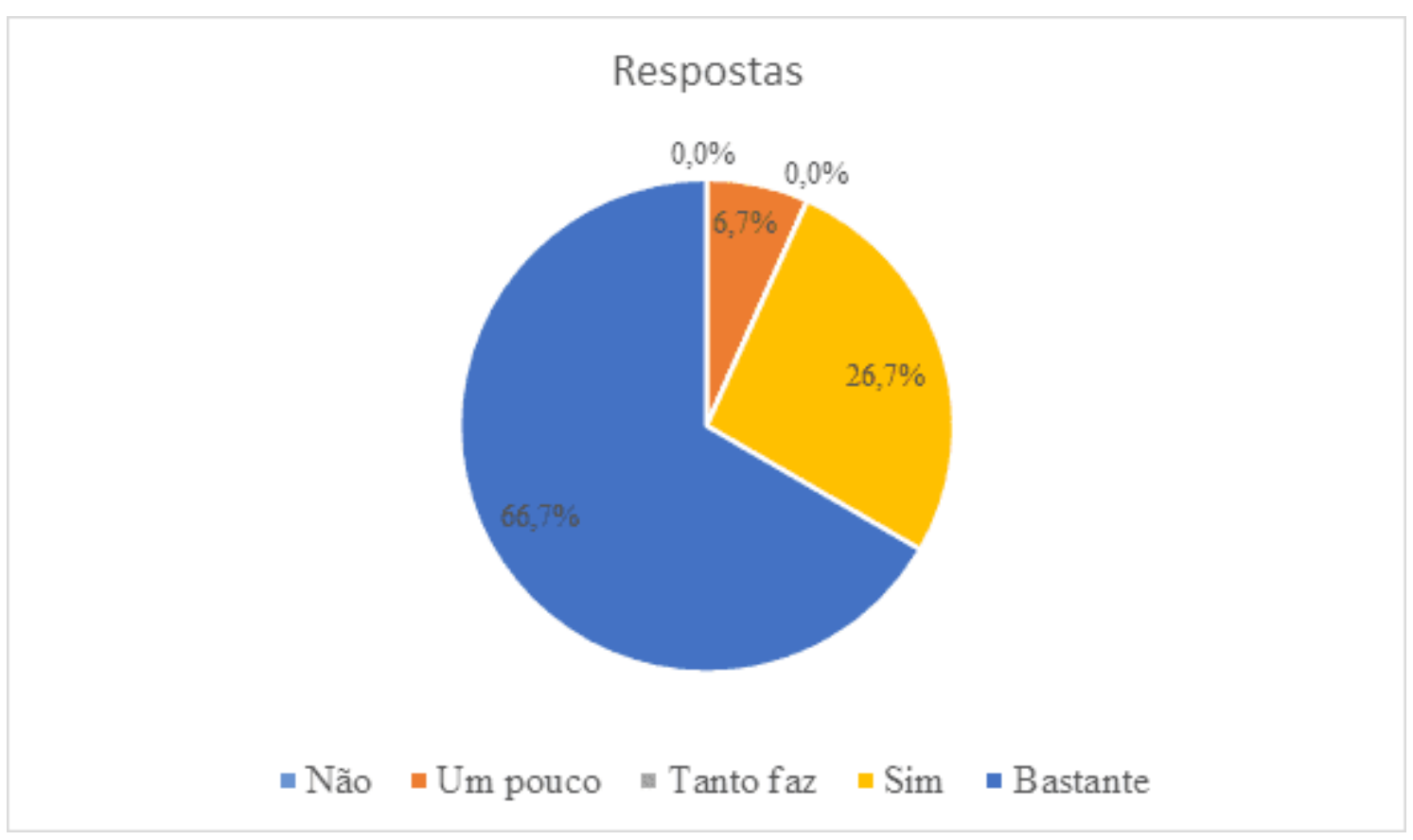

RC: 93729

Disponibile in: https://www.nucleodoconhecimento.com.br/formazione-it/cinematichecon-modellus 
Figura 15 - Domanda 2 della seconda fase (Autori, 2017)

2. Hai avuto difficoltà nel modo in cui il programma presenta i suoi risultati?

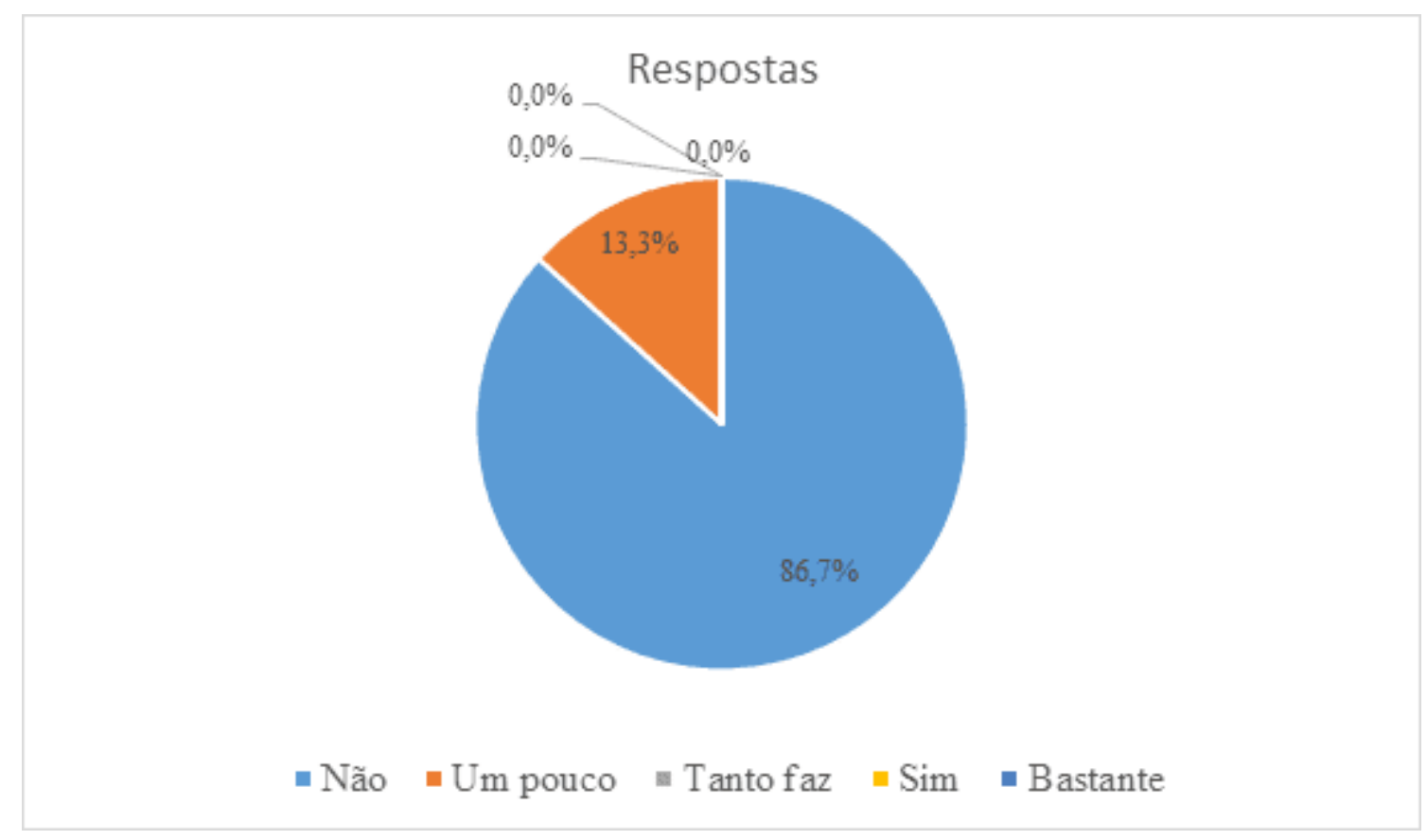

RC: 93729

Disponibile in: https://www.nucleodoconhecimento.com.br/formazione-it/cinematichecon-modellus 
Figura 16 - Domanda 3 della seconda fase (Autori, 2017)

3. Crede che l'uso di software come Modellus potrebbe essere utilizzato in classe per contribuire all'insegnamento della Fisica?

\section{Respostas}

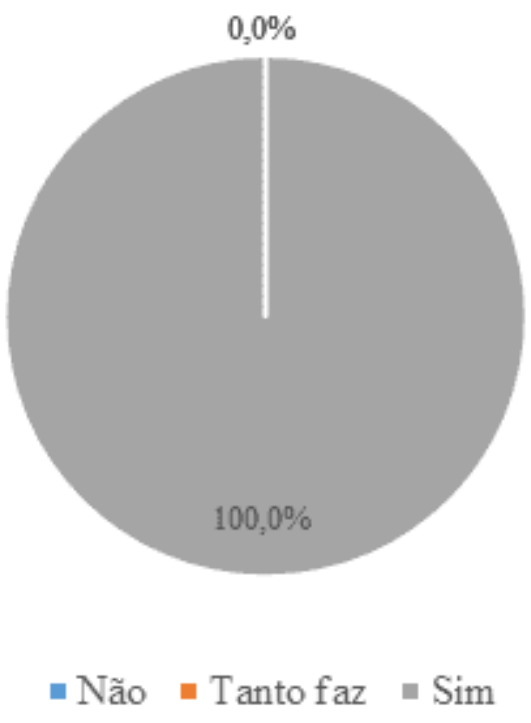

Si noti che le risposte erano molto positive riguardo al modellus come metodo di insegnamento, con una maggioranza che affermava che l'uso della modellazione computazionale facilitava la comprensione degli esercizi, anche se un po '. Quindi, solo una piccola parte presentava una certa difficoltà per quanto riguarda l'uso di Modellus, mentre la maggior parte non presentava una tale impresa.

Tutti hanno mostrato fiducia nell'uso del software come forma di insegnamento della fisica, con un'approvazione del $100 \%$. Si è conclusa con le considerazioni finali dei partecipanti, dove sono state presentate prospettive per l'applicazione di Modellus e suggerimenti per miglioramenti come modo per migliorare ulteriormente l'uso del programma:

4. Qual è la sua ultima considerazione del lavoro svolto?

- È una buona proposta implementare questo software nelle lezioni di fisica!

RC: 93729

Disponibile in: https://www.nucleodoconhecimento.com.br/formazione-it/cinematichecon-modellus 
- L'applicazione presentata è molto utile, in quanto ha come fine, per facilitare il processo di apprendimento in materie focalizzate sulla fisica, rendendo l'argomento più interattivo e dinamico.

- Un ottimo lavoro, sono orribile in fisica e onestamente ho imparato un argomento che ho trovato impossibile

- Il progetto presenta un modo diverso di vedere la fisica

Una grande risorsa.

- Molto buono e ricco per l'apprendimento

- Ho trovato il lavoro molto buono, perché ha notevolmente facilitato la comprensione degli esercizi proposti poiché la forma illustrativa di insegnamento facilita per gli studenti.

- Credo che il software Modellus dovrebbe essere implementato nelle lezioni di fisica

- L'applicazione è molto interessante, e sicuramente aiuterà nell'insegnamento e nell'apprendimento della disciplina della Fisica, essendo anche giocosa.

- Il lavoro del gruppo è stato quanto meno interessante. Considerando l'intenzione menzionata, lo sforzo applicato e soprattutto l'intenzione insieme alle loro azioni. II progetto merita, a mio avviso, un'attenzione particolare e tentativi per la sua applicazione in classe, perché la fisica (come scienza e disciplina) diventerà qualcosa di più complesso di quanto non sia senza una metodologia appropriata. Di conseguenza, aumenterà ulteriormente il "terrore" degli studenti al riguardo. Così, e, quindi, il lavoro del duo dovrebbe essere considerato come un grande "tentativo metodologico".

- È stato un lavoro ben fatto e facile da capire 
- Per quanto riguarda il programma, penso che ci deve essere più organizzazione nella separazione dei dati e delle icone di animazione. L'ho trovato molto interessante e potrei usarlo durante le lezioni. Soprattutto quando abbiamo bisogno di risultati rapidi. Un complimento per il programma è quando hanno usato le immagini nella rappresentazione dei risultati.

- Molto creativo e aiuta molto nell'interpretazione della domanda, penso che sia un buon strumento per l'uso in classe e nel lavoro.

- Il lavoro svolto è piuttosto interessante, e sarebbe di grande valore utilizzarlo in classe, in modo che noi studenti possiamo osservarlo in modo più esplicito.

- È un contributo all'insegnamento della fisica, aumentando ulteriormente la comprensione di varie materie, anche se necessita di miglioramenti in relazione al corretto utilizzo di questo software, credo che dovrebbe essere molto utile.

\section{CONSIDERAZIONI FINALI}

La ricerca educativa nel campo dell'insegnamento della fisica mostra che vi è un deficit nell'apprendimento, sia per difficoltà di disciplina, sia per metodologia degli insegnanti, materiali utilizzati, tra gli altri. L'uso delle tecnologie sarebbe un modo per compensare questo "fallimento" del sistema, poiché il campo tecnico dell'informazione è diventato molto vasto oggi, essendo utilizzato in vari settori della conoscenza umana. Da questo punto di vista, come soluzione, il software modellus viene portato come materiale per supportare l'insegnamento di concetti fisici, manipolando variabili, animazioni, tabelle e grafici.

Il programma è stato scelto proprio perché gratuito, e consente la visualizzazione dell'animazione e dei valori delle variabili e dei parametri utilizzati in esso. Si inserisce molto bene nell'ambito educativo, essendo utilizzato con l'obiettivo centrale di interpretare ciò che le quantità fisiche influenzano in un sistema, oltre allo sviluppo della conoscenza strutturale dei modelli attraverso equazioni che rappresentano il movimento di un oggetto, la direzione di un oggetto, ecc.

RC: 93729

Disponibile in: https://www.nucleodoconhecimento.com.br/formazione-it/cinematichecon-modellus 
Poiché il software funziona con i movimenti, era inevitabile sfuggire ai soggetti ricorrenti della cinematica, quindi, gli esercizi di lancio verticale e lancio obliquo sono stati presentati come un modo per testare il programma. È ricavato da studi di Veit e Teodoro (2002), Solano (2002), altri riferimenti e dal test stesso, che attraverso una semplice interfaccia, Modellus sviluppa il pensiero degli utenti e aiuta nell'insegnamento della Fisica con animazioni interattive. "Word Office" sul desktop.

Con il questionario condotto su Modellus utilizzando gli esercizi dei soggetti Vertical Launch e Oblique Release, è stato ottenuto nella prima valutazione che poco più del $75 \%$ dei valutati, non piace o piace alla fisica, avendo un cattivo rapporto con essa. È un fatto importante per la ricerca, poiché nella seconda parte, dopo aver mostrato le materie che utilizzano il programma, il $100 \%$ dei valutati ha risposto che il software è vantaggioso nell'apprendimento, mostrando come l'uso di altre metodologie, principalmente, l'utilizzo delle tecnologie possa essere un'ottima risorsa per l'insegnamento della fisica.

Modellus non è ancora ben noto in Brasile, forse per il bene del sistema educativo, che è considerato antico e basato su secoli passati da molti autori, che non regola molte forme diversificate di insegnamento per l'investimento delle aree. Questo potrebbe essere il problema di non utilizzare il programma, che, come notato, aiuta notevolmente nell'apprendimento delle scienze esatte, essendo affrontato in questo articolo l'area della Fisica. La soluzione per aumentare il numero di persone nell'istruzione del paese nelle classifiche mondiali potrebbe essere diversificare le metodologie utilizzate nell'insegnamento, e l'uso di Modellus sarebbe una pratica molto importante per questa esigenza.

\section{RIFERIMENTI}

ALIPRANDINI, Daiane Maria; SCHUHMACHER, Elcio; SANTOS, Muriel Clasen dos. Processo Ensino e Aprendizagem de Física apoiada em software de modelagem. In: I SIMPÓSIO NACIONAL DE ENSINO DE CIÊNCIA E 
TECNOLOGIA, 1., 2009, Ponta Grossa. Ciência e Tecnologia. Blumenau: Francisco; Junior; Pinheiro, 2009. p. 1370 - 1380.

ARAUJO, Ives Solano. Um Estudo sobre o Desempenho de Alunos de Física Usuários Da Ferramenta Computacional Modellus na Interpretação de Gráficos em Cinemática. 2002. 109 f. Dissertação (Mestrado) - Curso de Física, Instituto de Física, Universidade Federal do Rio Grande do Sul, Porto Alegre, 2002.

KRASILCHIK, M. Prática de ensino de biologia. 4. ed. São Paulo: Ed. da USP, p.198, 2004.

LIMA, Kênio Erithon Cavalcante; VASCONCELOS, Simão Dias. Análise da metodologia de ensino de ciências nas escolas da rede municipal de Recife. Ensaio: aval.pol.públ.Educ., Rio de Janeiro , v. 14, n. 52, p. 399,Set. 2006 . Disponível em:<http://www.scielo.br/scielo.php?script=sci_arttext\&pid=S0104$40362006000300008 \& \operatorname{lng}=e n \& n r m=i s o$ >. Acesso em: 05 out. 2017.

LOPES, Rosemara; FEITOSA, Eloi. Applets Como Recurso Pedagógico No Ensino De Física: Aplicação Em Cinemática. In: SIMPÓSIO NACIONAL DE ENSINO DE FÍSICA - SNEEF, 18., 2009, Vitória. Simpósio. Vitória: Unesp, 2009. p. $1-9$.

MIZUKAMI, Maria da Graça Nicoletti. Abordagem Tradicional. In: MIZUKAMI, Maria da Graça Nicoletti. Ensino: As abordagens do Processo. São Paulo: Epu, 1986. Cap. 1. p. 7-18. Disponível em: <https://www.docdroid.net/MrZCc0F/maria-dasgracas-nicoletti-mizukami-ensino-as-abordagens-do-processo. pdf\#page=14>.

Acesso em: 05 out. 2017.

PELIZZARI, Adriana; KRIEGL, Maria de Lurdes; BARON, Márcia Pirih; FINCK, Nelcy Teresinha Lubi; DOROCINSKI, Solange Inês. Teoria da Aprendizagem Significativa Segundo Ausubel. Pec, Curitiba, v. 2, n. 1, p.37-42, jul. 2002. 
RAMALHO JUNIOR, Francisco; FERRARO, Nicolau Gilberto; SOARES, Paulo Antônio de Toledo. Lançamento horizontal e lançamento oblíquo no vácuo. In: RAMALHO JUNIOR, Francisco; FERRARO, Nicolau Gilberto; SOARES, Paulo Antônio de Toledo. Os Fundamentos da Física 1: Mecânica. 9. ed. São Paulo: Moderna, 2007. Cap. 9. p. 144-162.

ROSA, Cleci Werner da; PEREZ, Carlos Ariel Samudio; DRUM, Carla. Ensino de Física nas Séries Iniciais: Concepções da Prática Docente. Revista Investigações em Ensino de Ciências (ienci), Porto Alegre, v. 12, n. 3, p.357-368, 09 out. 2009.

SANTOS, Gustavo H.; ALVES, Lynn; MORET, Marcelo A.. Modellus: Animações Interativas Mediando a Aprendizagem Significativa dos Conceitos de Física no Ensino Médio. Sitientibus: Série Ciências Físicas, Feira de Santana, v. 2, n. 7, p.56-67, nov. 2006. Anual. Disponível em: <http://dfis.uefs.br/sitientibus/vol2/Expediente2006_Main-SPSS.pdf>. Acesso em: 18 ago. 2017.

TEIXEIRA, Ricardo Roberto Plaza; JÚNIOR, Modesto Pantaleo; GOLFETTE, Bruno Henrique. PERFIL DOS PROFESSORES DE FÍSICA DO ENSINO MÉDIO EM SÃO PAULO. 2004. Disponível em: <http://www.ufpa.br/ensinofts/perfilpfsp.html>. Acesso em: 20 dez. 2005.

VEIT, E. A.; TEODORO, V. D.. Modelagem no Ensino: Aprendizagem de Física e os Novos Parâmetros Curriculares Nacionais para o Ensino Médio. Revista Brasileira de Ensino de Física, São Paulo, v. 24, n. 2, p.87-95, jul. 2002. Disponível em: <http://www.scielo.br/scielo.php?script=sci_arttext\&pid=S1806$11172002000200003 \&$ Ing=en\&nrm=iso >. Acesso em: 17 set. 2017.

Pubblicato: Settembre, 2019.

Approvato: Marzo, 2020. 